\title{
A Physically Based Cumulative Damage Formalism
}

\author{
Richard M. Christensen
}

\section{Lawrence Livermore National Laboratory and Stanford University}

\begin{abstract}
$\underline{\text { Abstract }}$
A general cumulative damage methodology is derived from the basic relation specifying crack growth rate (increment) as a power law function of the stress intensity factor. The crack is allowed to grow up to the point at which it becomes unstable, thereby determining the lifetime of the material under the prescribed stress program. The formalism applies for the case of creep to failure under variable stress history as well as for cyclic fatigue to failure under variable stress amplitude history. The formulation is calibrated by the creep rupture lifetimes at constant stress or the fatigue cycle lifetimes at constant stress amplitude. No empirical (non-physical) parameters are involved in the basic formulation; everything is specified in terms of experimentally determined quantities. Several examples are given showing the inadequacy of Linear Cumulative Damage while the present nonlinear damage accumulation method overcomes these deficiencies. The present method is extended to admit probabilistic conditions.
\end{abstract}




\section{$\underline{\text { Introduction }}$}

Damage accumulation in materials is very important, but very challenging to characterize in a meaningful and reliable manner. As the possible damage accumulates, the remaining lifetime under future loads becomes more limited. The ultimate goal is to be able to predict the remaining lifetime as the past history of loading induces a growing state of damage. More succinctly, the common purpose is to be given a complete loading spectrum and then predict how far into the loading sequence the material can remain coherent before suffering catastrophic failure.

The conditions under which these damage/lifetime conditions remain as the determining factor is creep failure conditions and fatigue conditions. The creep failure case corresponds to polymers under ambient and also high temperature conditions, as well as metals under high temperature conditions. The cyclic fatigue case corresponds to virtually all materials. The present investigation will consider both cases. Most of the derivation will be formed under the creep to failure condition and then it will be shown how through notational changes the results can be converted to the cyclic fatigue condition. Often the case of creep rupture is referred to as static fatigue.

The most common approach to such problems is to recognize that cracks under fatigue conditions usually grow in a manner with the rate of growth expressed as stress level (stress intensity factor) to some exponent. This is widely known as the Paris law, Paris [1], and has been verified for many materials over many decades of change on log scales. This power law form is then used to predict the number of load cycles until the crack reaches a pre-selected, unacceptable size. Such matters are discussed in two excellent sources, Suresh [2] and Kanninen and Popelar [3]. Particular models relate the rate of crack growth to nonlinear functions of the stress intensity factor. Such models include those of Wheeler [4], Willenborg et al. [5] and Elber [6]. Chudnovsky and Shulkin [7] give a somewhat different approach, which still results in typical lifetime forms. In a different approach, a strength evolution methodology has been developed by Reifsnider, most recently in Reifsnider and Case [2002]. The controlling form involves an integral representation with a power law time weighting function in the integrand. All of these models have a reasonable physical basis, and the work to be given here, although different, shares the same general background.

Another general approach is that of Linear Cumulative Damage, LCD. In this method increments of damage, expressed as fractions of lifetime at particular stress levels, are linearly added together to express total damage and thereby the lifetime. This method is also known as the Palmgren-Miner Law, Palmgren [9], Miner [10]. The method is completely empirical, but quite widely used because of its simplicity and utility. However, LCD is widely acknowledged to be inadequate. This is partially based upon its empirical nature and partly based upon its prediction of unsatisfactory results. LCD has been discussed by Stigh [11], referring to it as the Life-Fraction Rule. In particular LCD was shown to mathematically be related to the continuum damage formulation introduced by Kachanov [12], under certain special conditions. 
The present work is motivated by all of the approaches just described. In particular the power law form for crack growth will be used as providing a solid, physical approach for the method. The damage and life prediction forms will then be converted to integral forms superficially similar to those of LCD. However, detailed comparisons with LCD will be made in order to highlight its shortcomings and to display how the present methodology overcomes these shortcomings.

The starting point here will be that of work recently given by Christensen and Miyano [13]. This previous work had many of the features to be included in the present approach, but it did not successfully compare with typical data for creep rupture conditions in some ranges. In a second paper Christensen and Miyano [14] corrected this deficiency in data modeling, but only in the special case of creep rupture. The work to be given here, also corrects this deficiency, but does so more generally than just for the special case of creep rupture.

\section{$\underline{\text { Kinetic Crack Based Cumulative Damage and Life Prediction }}$}

Initially a state of growing damage is taken to be (highly) idealized as the self similar growth of a single crack. This idealization will be generalized later in the derivation. For the central crack problem start with the widely used power law form expressing the crack growth rate as a function of the stress intensity factor as

$$
\dot{a}=\lambda(\sigma \sqrt{a})^{r}
$$

where crack size a(t) and stress $\sigma(\mathrm{t})$ are functions of time, $\mathrm{r}$ is the power law exponent and $\lambda$ is a constant. Separate variables in (1) and integrate to get

$$
a^{1-\frac{r}{2}}(t)-a_{o}^{1-\frac{r}{2}}=\lambda\left(1-\frac{r}{2}\right) \int_{o}^{t} \sigma^{r}(\tau) d \tau
$$

where $a_{0}$ is the initial crack size and $\sigma(\tau)$ is the given stress history. Relation (1) is appropriate to the creep conditions that occur for polymers and for metals at high temperature.

Rewrite (2) as

$$
\left(\frac{a}{a_{o}}\right)^{1-\frac{r}{2}}-1=\lambda\left(1-\frac{r}{2}\right) a_{o}^{\frac{r}{2}-1} \int_{o}^{t} \sigma^{r}(\tau) d \tau
$$

The procedure here is to let the crack grow under the imposed load until it reaches the size at which it becomes unstable at the stress level that exists at that time. To carry out this process, a further relation between a(t) and $\sigma(\tau)$ is needed, which must come from the failure event. 
In the previous work, Christensen and Miyano [13], the critical stress intensity factor at time $t$ was used to provide the needed relationship. This then gave

$$
\sigma(\mathrm{t}) \sqrt{a(t)}=\sigma_{i} \sqrt{a_{o}}
$$

where $\sigma_{i}$ is the instantaneous static strength which characterizes failure for the virgin material with initial crack size $a_{0}$. Although this procedure is appealing, since it follows classical fracture mechanics lines, it did not yield entirely satisfactory results. The corresponding creep rupture times, at constant stress, did give the power law lifetime region controlled by exponent, $r$, but the transition region at higher stress levels than in the power law region did not provide a good match with data. This difficulty will be shown later as Eq. (17). At this point a more general procedure is needed than that followed previously.

Instead of using (4), take the crack size at failure instability as

$$
\frac{a}{a_{o}}=F\left(\frac{\sigma}{\sigma_{i}}\right), \text { at failure }
$$

where $F($ ) is some function yet to be determined. The form (5) rather than (4) is needed to generalize beyond the idealized single sharp crack in order to account for such effects as crack interaction, crack coalescence, complex non-ideal conditions at crack tips, other types of damage beyond that of idealized cracks such as de-bonding in the case of polymeric fiber composites, and a wide variety of other possible non-ideal effects. In the following work, the growing state of damage will continue to be referred to as that of a growing crack, but with the understanding this growing damage state may take a much more complicated form than that of the idealized single crack. Nevertheless, the basic kinetic relation (1) leading to (3) will be retained and used. The concept of the intrinsic static strength, $\sigma_{i}$, also will be retained and will play a pivotal role in the developments ahead.

Combining (3) and (5) gives

$$
1-F^{1-\frac{r}{2}}\left(\frac{\sigma}{\sigma_{i}}\right) \geq \lambda\left(\frac{r}{2}-1\right) a_{o}^{\frac{r}{2}-1} \int_{o}^{t} \sigma^{r}(\tau) d \tau
$$

The inequality in (6) applies for times less than the failure time wherein the crack has not yet reached the critical size for failure. The equality in (6) is at the time then given by $t$.

It is convenient to express (6) in terms of non-dimensional variables. Let nondimension stress be given by

$$
\tilde{\sigma}=\frac{\sigma}{\sigma_{i}}
$$


and non-dimensional time by

$$
\tilde{t}=\frac{t}{t_{1}}
$$

where

$$
t_{1}=\frac{1}{\lambda\left(\frac{r}{2}-1\right) a_{o}^{\frac{r}{2}-1} \sigma_{i}^{r}}
$$

Henceforth $t_{1}$ will be treated as a quantity to be determined from data. On $\log \tilde{\sigma}$ versus $\log \tilde{t}$ scales different values of $\sigma_{i}$ gives shifts in the vertical direction while $\mathrm{t}_{1}$ gives shifts in the horizontal direction.

With (7)-(9) the form (6) becomes

$$
1-F^{1-\frac{r}{2}}(\tilde{\sigma}) \geq \int_{o}^{\tilde{t}} \tilde{\sigma}^{r}(\tau) d \tau
$$

Now, let

$$
f(\tilde{\sigma})=1-F^{1-\frac{r}{2}}(\tilde{\sigma})
$$

giving (10) as

$$
\int_{o}^{\tilde{t}} \tilde{\sigma}^{r}(\tau) d \tau \leq f(\tilde{\tilde{\sigma}}(\tilde{t}))
$$

where the equality applies at the failure event giving the lifetime. Determine $f(\tilde{\sigma})$ from the basic creep rupture behavior at constant stress. Take the given spectrum of creep rupture life times as

$$
\tilde{t}=\tilde{t}_{c}(\tilde{\sigma}), \text { at constant stress }
$$

Using (13) in (12) at equality gives $f(\tilde{\sigma})$ as

$$
f(\tilde{\sigma})=\tilde{\sigma}^{r} \tilde{t}(\tilde{\sigma})
$$

As the final step in this procedure, substitute (14) into (12) and write the inequality and equality in separate forms as 


$$
\begin{aligned}
& \underline{t}<\text { Lifetime } \\
& \underbrace{\int_{0}^{\tilde{t}} \tilde{\sigma}^{r}(\tau) d \tau}_{\substack{\text { Actual crack size } \\
\text { at time } \tilde{\mathrm{t}}}}<\underbrace{\tilde{\left.\sigma^{r}(\tilde{t}) \tilde{t_{c}}(\tilde{\tilde{\sigma}} \tilde{t})\right)}}_{\begin{array}{c}
\text { Crtical crack size for } \\
\text { stress at time } \tilde{\mathrm{t}}
\end{array}} \\
& \tilde{t}=\text { Lifetime } \\
& \int_{0}^{\tilde{t}} \tilde{\sigma}^{r}(\tau) d \tau=\tilde{\sigma}^{r}(\tilde{t}) \tilde{t}_{c}(\tilde{\sigma}(\tilde{t}))
\end{aligned}
$$

As noted under (15) the actual crack size has not yet reached the critical size. Thereafter, the crack does reach the critical size and the lifetime $\tilde{t}$ is determined from (16). Next the problem of determining exponent $r$ in (16) is taken up. Rarely does one have direct data on the crack growth rate for a particular material. It would be advantageous to be able to determine r directly from $\tilde{t}_{c}(\tilde{\sigma})$.

If instead of the procedure just developed, the simplified expression (4) had been used, the resulting creep function would have been found to be given by

$$
\tilde{t}_{c}=\frac{1}{\tilde{\sigma}^{r}}-\frac{1}{\tilde{\sigma}^{2}}
$$

This result from Christensen and Miyano [13] gives the power law range result for low stress as

$$
\tilde{t}_{c} \rightarrow \frac{1}{\tilde{\sigma}^{r}}
$$

however, (17) does not otherwise give a good fit with data. Thus in this case, the power law exponent in the kinetic crack expression (1) is the same as the power law exponent in the lifetime expression (18). The more general result (16) is still taken to have this same behavior such that $r$ in (16) corresponds to the inverse slope in the lifetime power law region, when it exists as in Fig. 1a. The case in Fig. 1a is now generalized to the case of a power law region as in Fig. 1b. Finally the form in Fig. 1c with an inflection point is simply the limiting case of Fig. 1b type. Thus exponent $r$ in (16) is determined by the inverse slopes in power law regions as shown in Fig. 1a, 1b, and 1c. 
The cumulative damage relation (15) and the lifetime relation (16) along with the determination of $\mathrm{r}$ from $\tilde{t}_{c}(\tilde{\sigma})$ as shown in Fig. 1 are the main results of this derivation. It is to be expected that the critical crack size at time $\tilde{t}$ in (16) depends upon the stress level at that time. Lower stress levels require larger crack sizes.

There is a simple conceptual test that can be applied to the result (16). As discussed by Christensen and Miyano [13] the lifetime results for constant strain rate testing, CSR, gives lifetimes that are shifted on log scales from those for creep rupture. To test this behavior on (16) take the stress history as

$$
\tilde{\sigma}=\beta \tau
$$

where $\beta$ is a constant. Substitute this into the equality form of (12), and integrate to get

$$
\tilde{t}^{\mathrm{r}+1}=\frac{r+1}{\beta^{r}} f(\tilde{\sigma}(\tilde{t}))
$$

Now eliminate $\beta$ from (20) using (19) to get

$$
\tilde{t}=(r+1) \frac{f(\tilde{\sigma}(\tilde{t}))}{\tilde{\sigma}^{r}(\tilde{t})}
$$

From (14) this can be written as

$$
\tilde{t}=(r+1) \tilde{t}_{c}(\tilde{\sigma})
$$

Thus using (22) the lifetime for CSR can be written as

$$
\log \tilde{t}_{C S R}=\log \tilde{t}_{c}+\log (r+1)
$$

The shifting property relating creep rupture and CSR to failure is found to be preserved by the general form (16).

At this point it is useful to compare the present cumulative damage result (16) with that from linear cumulative damage, LCD. The LCD form is

$$
\int_{o}^{\tilde{t}} \frac{d \tau}{t_{c}(\tilde{\sigma}(\tau))}=1
$$

whereas the present form, rewritten from (16) is 


$$
\frac{1}{\tilde{\sigma}^{r}(\tilde{t}) \tilde{t}_{c}(\tilde{\sigma}(\tilde{t}))} \int_{o}^{\tilde{t}^{\tau} \tilde{\sigma}^{r}(\tau) d \tau=1}
$$

Note that if the front factor in (25) were arbitrarily moved to inside the integral at time $\tau$, the result would be identically that of LCD, (24). But there is no justification for this transposition, it would destroy the kinetics inherent in the present derivation. LCD, (24), can only be viewed as a directly postulated damage relation which is totally empirical. Lifetime relation (25) follows from the physical derivation given here.

\section{$\underline{\text { A Special Form }}$}

There is a special form for creep rupture $\tilde{t}_{c}(\tilde{\sigma})$ of the type shown in Fig. 1a that affords considerable advantage in applications, Christensen and Miyano [14]. This is given by

$$
\tilde{t}_{c}=\frac{\left(1-\tilde{\sigma}^{p}\right)^{q}}{\tilde{\sigma}^{r}}
$$

where $\mathrm{p}, \mathrm{q}$, and $\mathrm{r}$ are parameters, with $\mathrm{r}$ being that for the power law range. With (26) the form (16) becomes

LCD is given by

$$
\frac{1}{\left[1-\tilde{\sigma}^{p}(\tilde{t})\right]^{q}} \int_{o}^{\tilde{t} \tilde{\sigma}^{r}(\tau) d \tau=1}
$$

$$
\int_{o}^{\tilde{t}} \frac{\tilde{\sigma}^{r}(\tau) d \tau}{\left[1-\tilde{\sigma}^{p}(\tau)\right]^{q}}=1
$$

As a first application of the present results and LCD consider the case of residual instantaneous strength after the loading of the material for a specified amount of time. The problem is as shown in Fig. 2. The material is loaded at stress $\tilde{\sigma}_{1}$ for time $\tilde{t}_{1}$ which is less than that which would cause creep rupture, but nevertheless does impart some damage to the material. Relation (27) then gives 


$$
\frac{1}{\left(1-\tilde{\sigma}_{R}^{p}\right)^{q}}\left(\tilde{\sigma}_{1}^{r} \tilde{t}_{1}\right)=1
$$

where $\tilde{\sigma}_{R}$ is the residual strength. Solving (29) for $\tilde{\sigma}_{R}$ gives

$$
\tilde{\sigma}_{R}=\left(1-\tilde{\sigma}_{1}^{\frac{r}{q}} \tilde{t}_{1}^{\frac{1}{q}}\right)^{\frac{1}{p}} \quad, \text { Present }
$$

It is easiest to reason the LCD result directly from (24). Up to time $\tilde{t_{1}}$ in Fig. 2 the integral has some value say $\alpha$ which is less than one. The following instantaneous loading to $\tilde{\sigma}_{R}$ must generate a value $1-\alpha$ to satisfy the equality in (24). For this integral to have a finite value over a vanishing time interval requires that $\tilde{t}_{c}\left(\tilde{\sigma}_{R}\right)=0$. This latter result only occurs for $\tilde{\sigma}_{R}=1$, so

$$
\tilde{\sigma}_{R}=1, \text { LCD }
$$

or

$$
\sigma_{R}=\sigma_{i}
$$

As a simple example take

$$
\begin{aligned}
& p=15 \\
& q=100 \\
& r=10
\end{aligned}
$$

The resulting creep rupture lifetime function is shown in Fig. 3. Its time variation is spread over many decades, which is typical for many materials. At $\tilde{\sigma}_{1}=0.5$ and $t_{1}=\frac{1}{2} \tilde{t}_{c}\left(\tilde{\sigma}_{1}\right)$ it is found from $(30)$ that

while

$$
\tilde{\sigma}_{R}=0.718, \text { Present }
$$

$$
\tilde{\sigma}_{R}=1 \quad, \quad \text { LCD }
$$

Thus the present residual strength prediction is considerably above the previous load level, but still much less than $\tilde{\sigma}=1$. In contrast LCD is unable to account for the previous accumulation of damage, and simply predicts the undamaged value of $\tilde{\sigma}_{R}=1$. LCD is extremely unconservative in this case.

\section{Cyclic Fatigue}


The previous results for creep damage type of cumulative effect can be converted to the case of cyclic fatigue by suitable notation and terminology changes.

Let $\mathrm{f}$ be the frequency in cycles per unit time and $\mathrm{n}$ be the number of cycles. Then the elapsed time is

$$
t=\frac{n}{f}
$$

Some materials show a frequency dependence, so the present method will be developed for fixed frequency and fixed form of the variation within one cycle. The starting point is to define and take as given the standard fatigue relation between constant stress $\tilde{\sigma}$ and the number of cycles to failure. Write this as

$$
\mathrm{n}=\mathrm{N}(\tilde{\tilde{\sigma}}), \text { constant stress }
$$

Where $\mathrm{n}$ is the cycles to failure and $\tilde{\sigma}$ is the non-dimensional stress

$$
\tilde{\sigma}=\frac{\sigma}{\sigma_{i}}
$$

where $\sigma_{i}$ is the instantaneous static strength corresponding to the maximum tensile stress within the cycle of variation.

With these notational changes the previous lifetime creep damage form (16) converts to the cyclic fatigue case as

$$
\int_{o}^{n} \tilde{\sigma}^{r}(\eta) d \eta=\sigma^{r}(n) N(\tilde{\sigma}(n))
$$

where stress is allowed to have a variable amplitude so long as the wave form and frequency are unchanged. Variable $\eta$ in (35) is the past history variable for $n$. Exponent $r$ in (35) is determined by the same method as in the creep damage case of Fig. 1. Any of the three forms in Fig. 1 are admissible with the case of Fig. 1a illustrated in Fig. 4.

The term on the left side of (35) represents the current crack size while the term on the right side is the critical size at the stress existing at $\mathrm{n}$ number of cycles. The equality in (35) is for the lifetime $\mathrm{n}$ in number of cycles.

\section{$\underline{\text { Probabilistic Generalization }}$}

For extension to probabilistic conditions the terminology of the creep damage case will be used. It will be understood that the same extension applies to fatigue conditions through the change of notation given in the previous section. 
Rewrite the deterministic form (16) for the creep case as

$$
\int_{o}^{\tilde{t}}\left[\frac{\sigma(\tau)}{\sigma_{i}}\right]^{r} d \tau=\left[\frac{\sigma(\tilde{t})}{\sigma_{i}}\right]^{r} t_{c}\left(\frac{\sigma(\tilde{t})}{\sigma_{i}}\right)
$$

The instantaneous static strength, $\sigma_{i}$, will be generalized to a probabilistic form through any particular distribution function. With this form then $\sigma_{i}$ can be written as

$$
\sigma_{i}=\phi(k)
$$

where $\phi(k)$ is a function of the quantile of failure, $\mathrm{k}, 0 \leq \mathrm{k} \leq 1$. Function $\phi(k)$ is determined by the distribution function, such as a Weibul form or any other one. Let the probabilistic, non-dimensional time to failure be given by $\zeta$. Now, the probabilistic form of (36) is given by

$$
\int_{o}^{\xi}\left[\frac{\tilde{\sigma}(\tau)}{\phi(k)}\right]^{r} d \tau=\left[\frac{\tilde{\sigma}(\zeta)}{\phi(k)}\right]^{r} \tilde{t}_{c}\left(\frac{\tilde{\sigma}(\zeta)}{\phi(k)}\right)
$$

Given the stress history and $\mathrm{k}$, this relation can be solved for the probabilistic time to failure, $\zeta$.

The result is particularly simple for the creep rupture case itself. Write the resulting probabilistic time to failure $\xi$ as a function of the applied stress level and $\phi(k)$. Then

$$
\zeta=h\left(\frac{\tilde{\sigma}}{\phi(k)}\right)
$$

Now use log scales for stress and time giving

$$
\log \zeta=g(\log \tilde{\sigma}-\log \phi(k))
$$

Where $\mathrm{g}(\mathrm{)})$ is a function found from $\mathrm{h}(\mathrm{)}$ ) after the conversion to log scales. This form reveals a vertical shift along the $\log \tilde{\sigma}$ axis with all curves emanating from a single master curve.

The above procedure was illustrated by Christensen and Miyano [13] for the case of using Weibul statistics to represent $\sigma_{i}$. The resulting lifetimes can be shown to be of Weibul form within the power law range but they are not of Weibul form outside of it. 
Christensen and Miyano [14] provided further experimental verification for these related forms.

\section{Examples}

Several examples will be given to illustrate the difference between the present approach and LCD. The creep rupture form given by (26) will be used with parameter values in (32). Examples of two and three stress step levels will be used.

For two stress levels as shown in Fig. 5, the present form (27) gives

$$
\tilde{t}=\tilde{t}_{1}+\frac{\left(1-\tilde{\sigma}_{2}{ }^{p}\right)^{q}}{\tilde{\sigma}_{2}{ }^{r}}-\tilde{t}_{1}\left(\frac{\tilde{\sigma}_{1}}{\tilde{\sigma}_{2}}\right)^{r}
$$

For LCD form (28) gives

$$
\tilde{t}=\tilde{t}_{1}+\frac{\left(1-\tilde{\sigma}_{2}{ }^{p}\right)^{q}}{\tilde{\sigma}_{2}{ }^{r}}-\tilde{t}_{1}\left(\frac{\tilde{\sigma}_{1}}{\tilde{\sigma}_{2}}\right)^{r}\left(\frac{1-\tilde{\sigma}_{2}^{p}}{1-\tilde{\sigma}_{1}^{p}}\right)^{q}
$$

The last term in parenthesis in (41) is the only difference in the two expressions. In some cases it will be found to be a crucial difference. Three step examples are also readily formed from (27) and (28). The specific examples are as follows.

\section{Example 1}

$$
\begin{aligned}
& \tilde{\sigma}_{1}=0.5 \text { up to } \tilde{t}_{1}=500 \\
& \text { then } \\
& \tilde{\sigma}_{2}=0.7 \text { to failure }
\end{aligned}
$$

Lifetimes:

$$
\begin{aligned}
& \tilde{t}=504.7 \text { Present } \\
& \tilde{t}=511.2 \mathrm{LCD}
\end{aligned}
$$

\section{Example 2}

$$
\begin{aligned}
& \tilde{\sigma}_{1}=0.85 \text { to } \tilde{t}_{1}=0.0005 \\
& \text { Then } \\
& \tilde{\sigma}_{2}=0.5 \text { to failure }
\end{aligned}
$$


Lifetimes:

$\tilde{t}=1020.8$ Present

$\tilde{t}=83.5 \mathrm{LCD}$

Example 3

$\tilde{\sigma}_{1}=0.5$ to $\tilde{t}_{1}=500$

Then

$\tilde{\sigma}_{2}=0.7$ to $\tilde{t}_{2}=504$

Then

$\tilde{\sigma}_{1}=0.5$ to failure

Lifetimes:

$\tilde{t}=909.2$ Present

$\tilde{t}=839.2 \mathrm{LCD}$

Example 4

$\tilde{\sigma}_{1}=0.5$ to $\tilde{t}_{1}=10$.

Then

$\tilde{\sigma}_{2}=0.8$ to $\tilde{t}_{2}=10.15$

Then

$\tilde{\sigma}_{1}=0.5$ to Failure

Lifetimes:

$\tilde{t}=1004.5$ Present

$\tilde{t}=430.1 \mathrm{LCD}$

These examples show significant differences between the present method and LCD. It is not easy to prescribe simple rules by which LCD is or is not acceptable except to say that for increasing loads the LCD results appear to be satisfactory.

\section{Extended Life Examples}

Now some examples will be given for which the LCD results exhibit the most egregious shortcomings. The examples are for cases where at a constant load the material is taken right up to the point of incipient failure. But just an instant before 
failure the load is reduced to a specified level. The problem is to determine the remaining lifetime at the reduced load level.

Use the same material specification through $\tilde{t}_{c}(\tilde{\sigma})$ in (26) with parameter values in (32). Take $\tilde{\sigma}_{1}=0.6$ up to incipient failure and then reduce the stress levels to $\tilde{\sigma}_{2}=0.5$, 0.4 , or 0.3 and find the remaining total lifetimes. At $\tilde{\sigma}=0.6$ the creep rupture lifetime is

$$
\tilde{t}_{c}=157.8
$$

Using expression (40) the present lifetime extensions are found as

$$
\begin{aligned}
& \tilde{\sigma}_{2}=0.5, \tilde{t}=\tilde{t}_{c}+\Delta \tilde{t}=201.7 \\
& \tilde{\sigma}_{2}=0.4, \tilde{t}=594.9 \\
& \tilde{\sigma}_{2}=0.3, \tilde{t}=7,938.0
\end{aligned}
$$

In the third case the lifetime is extended by a factor of 50 .

When the load level is taken to incipient failure, the LCD method predicts that any additional load at any level will produce immediate failure, as can be verified from (41) with $\tilde{t}_{1}=\tilde{t}_{c}$. Thus for LCD

$$
\tilde{\Delta t}=0
$$

LCD is unable to account for the fact that the reduced stress level requires that the crack obtain an increasing size before it can reach the criticality condition at the reduced stress level.

\section{$\underline{\text { Conclusions }}$}

The main results of the present work are the lifetime forms (16) for creep failure and (35) for fatigue. Exponent $\mathrm{r}$ in (16) is evaluated from creep rupture at constant stress $\tilde{t}_{c}(\tilde{\sigma})$ as shown in Fig. 1, with a similar operation for the case of fatigue. These forms accommodate prescribed variable stress amplitudes, but in the case of fatigue the frequency and wave forms are taken to be unchanged. In the non-dimensional stress (7) and non-dimensional time (8), $\sigma_{i}$ and $t_{1}$ are calibrating factors that allow vertical and horizontal shifts along the $\log$ axes. The basic failure property inputs are the creep 
rupture life times $\tilde{t}_{c}(\tilde{\sigma})$ in (16) and fatigue lifetimes $\mathrm{N}(\tilde{\sigma})$ in (35) both at constant stress amplitude. There are no empirical parameters involved in the theory and final lifetime forms.

Several conclusions can be drawn from the general structure of the theory and the examples that have been considered. Comparing LCD with the present more complete and physically based methodology shows that

(i) LCD is acceptable for monotonically increasing loads (stress).

(ii) LCD is not satisfactory for the residual strength problem. After a given stress history, but before failure, the LCD residual instantaneous static strength, $\tilde{\sigma}_{R}$ is undiminished from the virgin material value of $\tilde{\sigma}_{R}=1$. The present method gives a reduced value for $\tilde{\sigma}_{R}$ based upon the damage accumulated in the past stress history.

(iii) The following conclusions apply for the extended life problem where the stress history is taken up to incipient failure, but instantaneously reduced to a lower stress level in order to prolong the life of the material. LCD gives no additional or extended lifetime after the decrease in load level. The present method gives an extended lifetime beyond the point at which the load is suddenly reduced. This is perhaps the most fundamental difference between the two methods with LCD being unrealistically conservative.

In the other examples that have been considered, some cases showed a good comparison between the two methods while others showed a poor comparison, sometimes different by an order of magnitude or more. In general it must be said that LCD gives an unacceptable result in an unacceptable number of cases.

The general conclusion is that this new cumulative damage formalism is no more difficult to apply than Linear Cumulative Damage, but it has a physical interpretation based upon crack growth and it readily admits generalization to probabilistic conditions. 


\section{$\underline{\text { References }}$}

[1] Paris P. C., The Growth of Cracks Due to Variations in Loads, PhD Thesis, Lehigh University, 1960.

[2] Suresh S., Fatigue of Materials, $2^{\text {nd }}$ Ed. Cambridge Univ. Press, 1998.

[3] Kanninen M. F. and Poplar C. H., Advanced Fracture Mechanics, Oxford Univ. Press, 1985.

[4] Wheeler O. E., "Spectrum Loading and Crack Growth", J. Basic Engineering 1972; 94:181-185.

[5] Willenborg J., Engle R. M. and Wood H., "A Crack Growth Retardation Model Using an Effective Stress Intensity Concept", Technical Report TFR 71-701 North American Rockwell, 1971.

[6] Elber W., Fatigue Crack Closure Under Cyclic Tension", Engineering Fracture Mechanics 1970; 2:37-45.

[7] Chudnovsky A. and Shulkin Y., "A Model for Lifetime and Toughness of Engineering Thermoplastics", in Inelasticity and Damage in Solids Subject to Microstructural Change, edited by I. J. Jordan, R. Seshadri and I. L. Meglis Memorial Press, Univ. Newfoundland, 1996.

[8] Reifsnider K. L. and Case S. C., Damage Tolerance and Durability in Material Systems 2002; Wiley-Interscience.

[9] Palmgren A., "Die Lebensdauer von Kugellagren" Zeitscrift des Vereins Deutscher Ingenieure $1924 ;$ 68:339-164.

[10] Miner M. A., "Cumulative Damage in Fatigue" J. Applied Mechanics 1945; 12: 159-164.

[11] Stigh U., "Continuum Damage Mechanics and the Life-Fraction Rule", J. Applied Mechanics 2006; 73: 702-704.

[12] Kachanov E. L., "On the Time to Failure Under Creep Conditions", Izv. Akad. Nauk SSR, Otd Tekhn. Naukn. Nauk 1958; $\underline{8}:$ 26-31.

[13] Christensen R. M. and Miyano Y., "Stress Intensity Controlled Kinetic Crack Growth and Stress History Dependent Life Prediction with Statistical Variability", Int. J. Fracture 2006; 137: 77-87. 
[14] Christensen R. M. and Miyano Y., "Deterministic and Probabilistic Lifetimes From Kinetic Crack Growth-Generalized Forms", Int. J. Fracture (in press). 

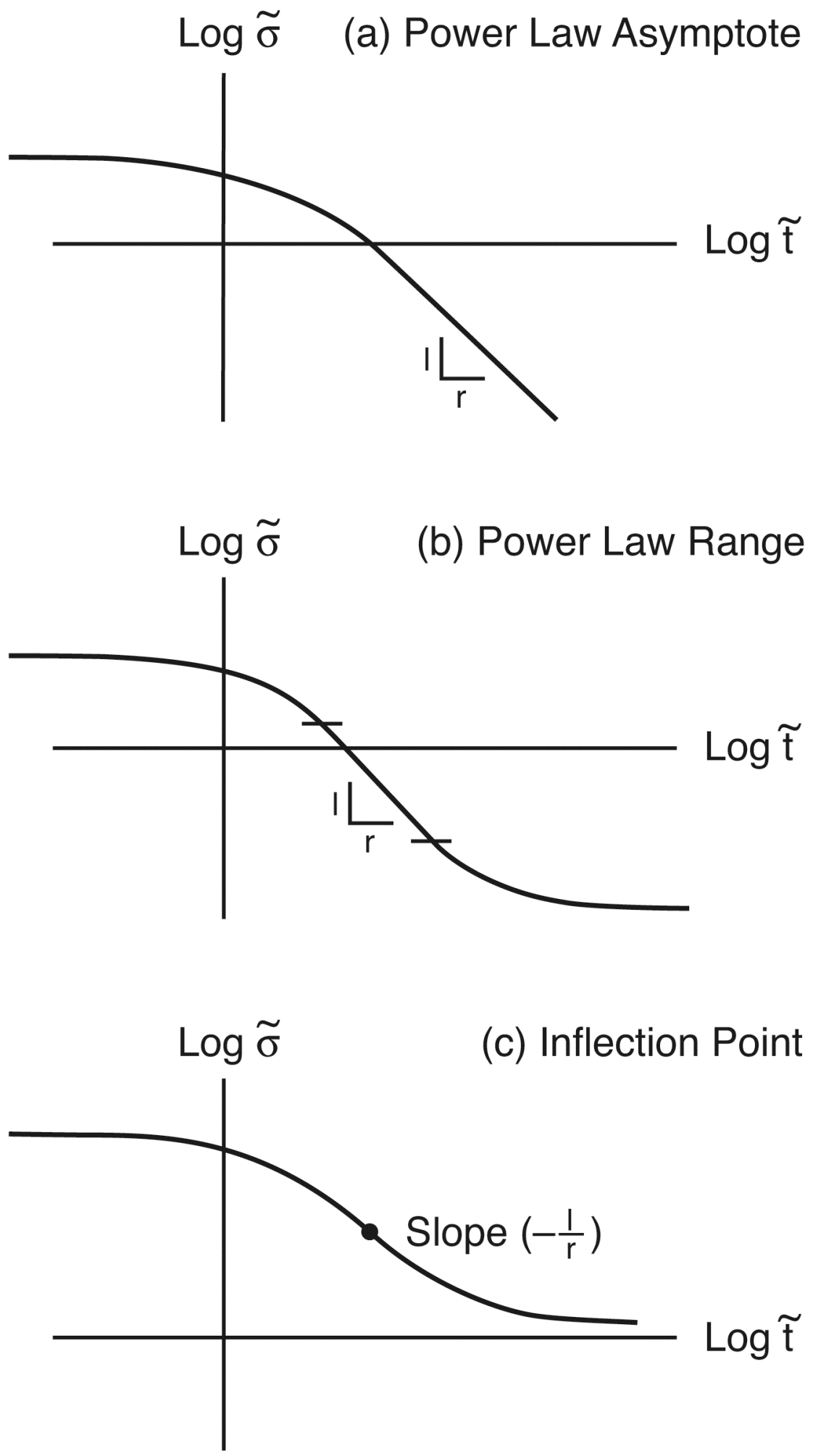

Figure 1. Determination of "r" From Creep Rupture 


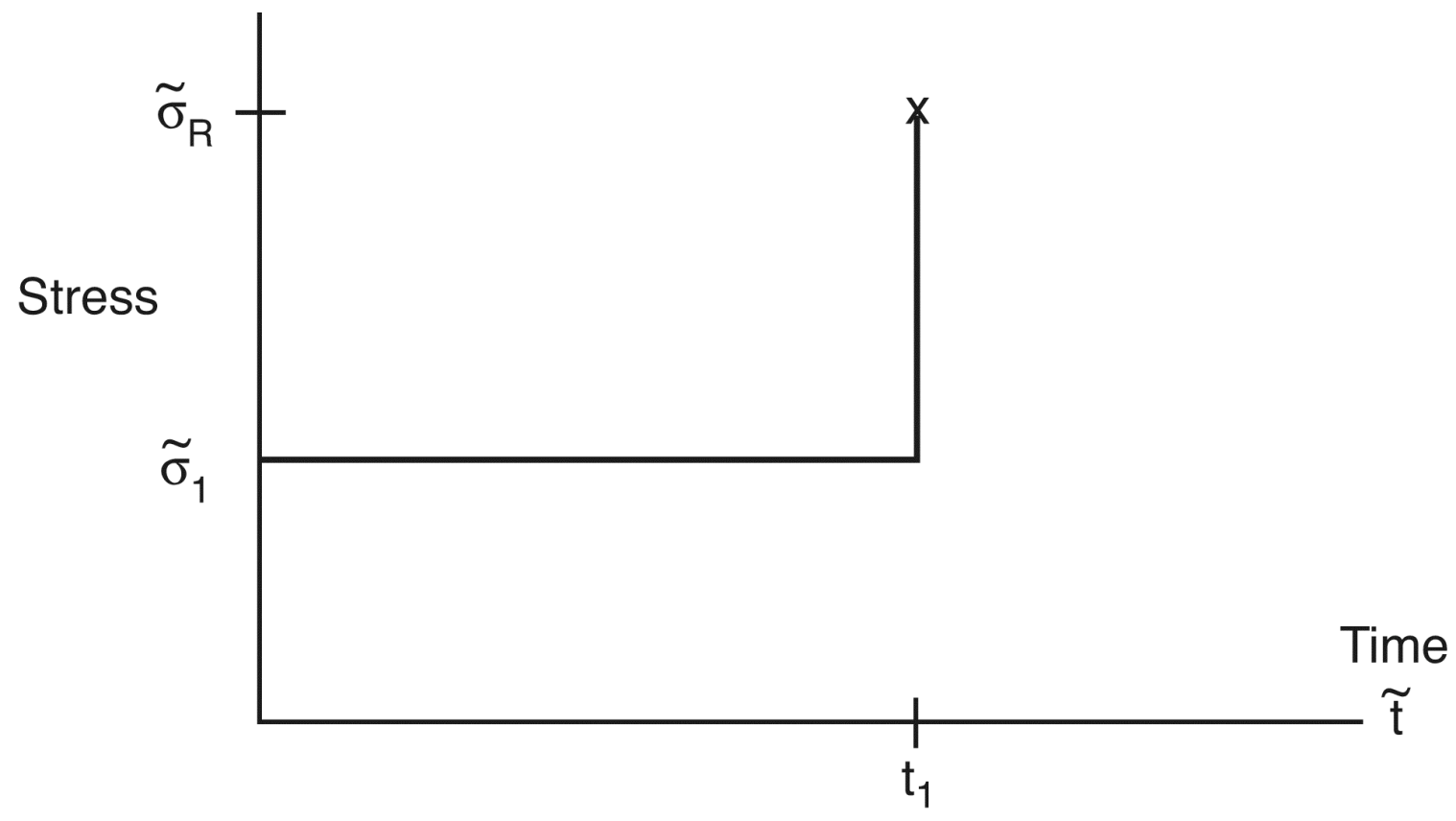

Figure 2. Instantaneous Residual Strength 


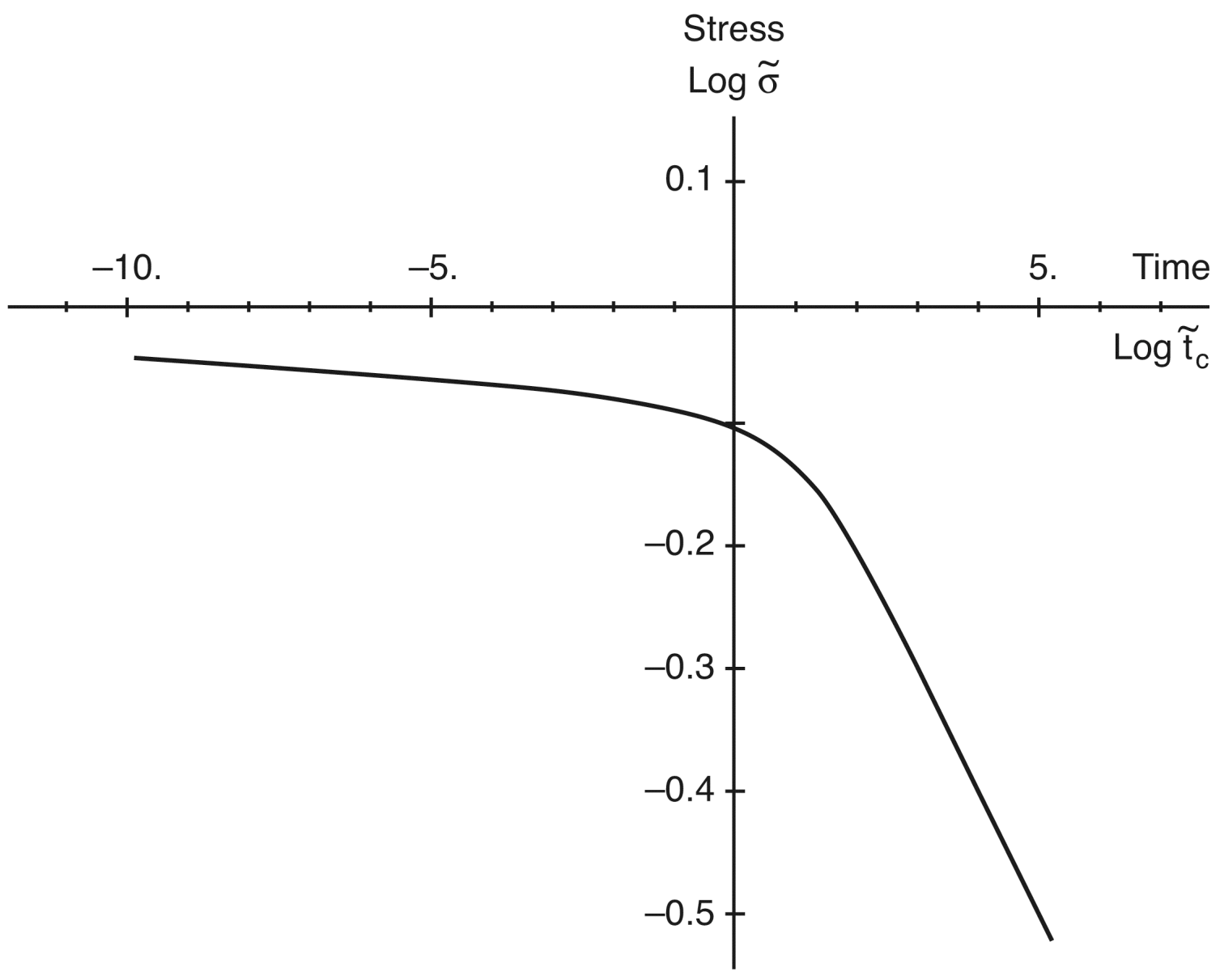

Figure 3. Creep Rupture Example, Eq. (26): $\mathrm{p}=15, \mathrm{q}=100$, and $\mathrm{r}=10$ 


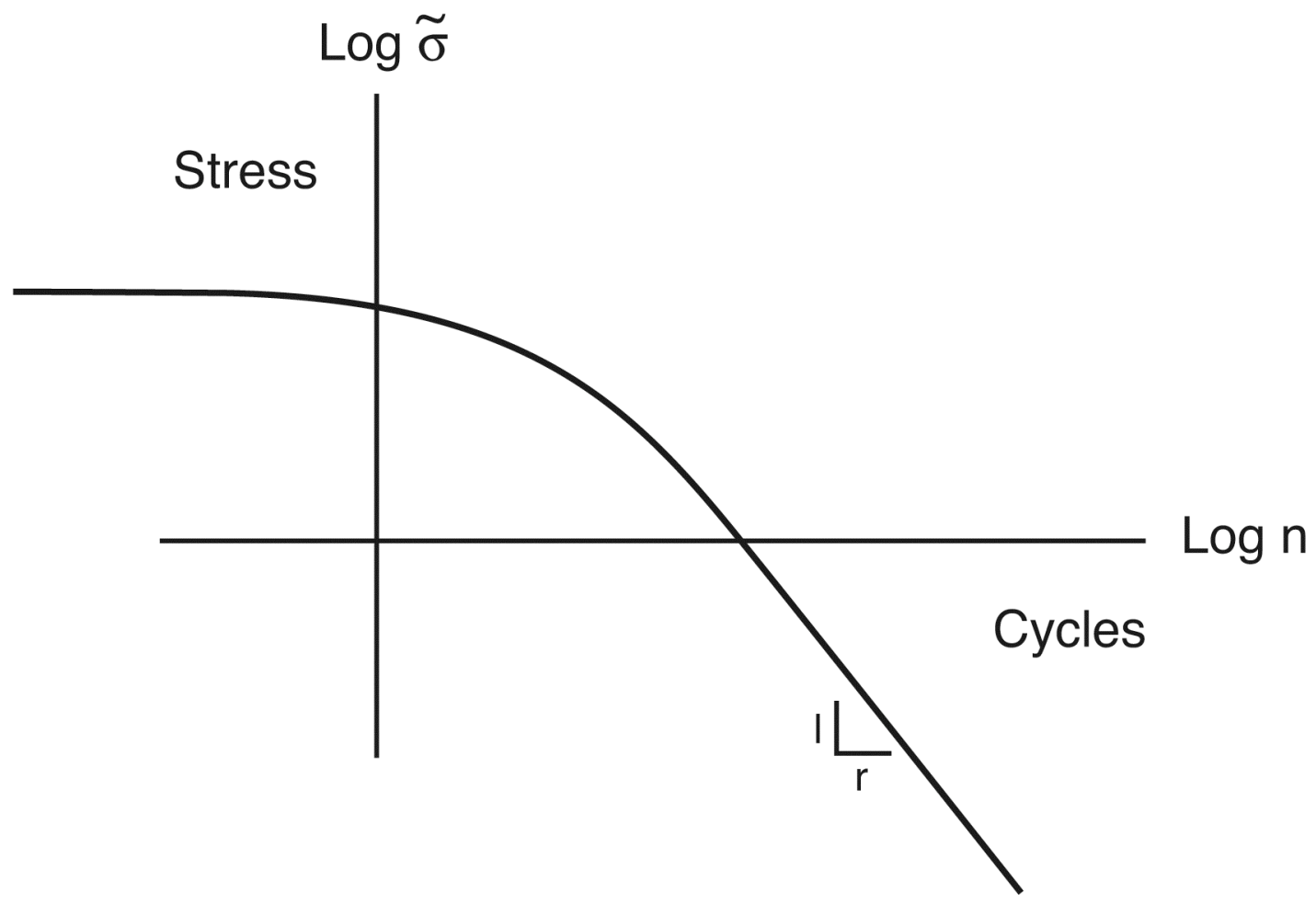

Figure 4. Fatigue Counterpart of Figure 1 


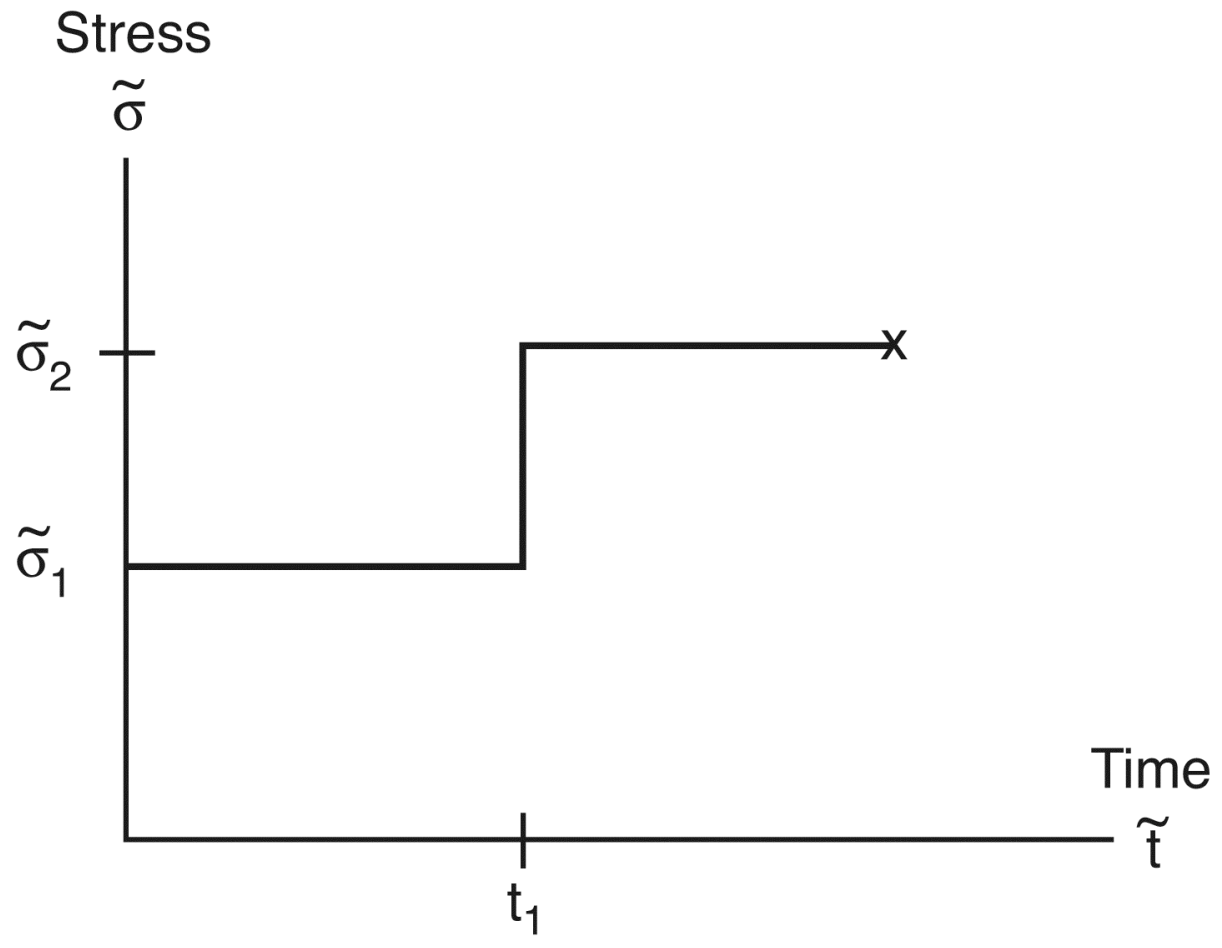

Figure 5. Two Step Load 